\title{
Systems Biology and Mechanistic Explanation
}

\author{
Ingo Brigandt, ${ }^{1}$ Sara Green ${ }^{2}$ and Maureen A. O'Malley ${ }^{3}$ \\ 1. University of Alberta \\ 2. University of Copenhagen \\ 3. University of Bordeaux
}

\begin{abstract}
We address the question of whether and to what extent explanatory and modelling strategies in systems biology are mechanistic. After showing how dynamic mathematical models are actually required for mechanistic explanations of complex systems, we caution readers against expecting all systems biology to be about mechanistic explanations. Instead, the aim may be to generate topological explanations that are not standardly mechanistic, or to arrive at design principles that explain system organization and behaviour in general, but not specific mechanisms. These abstraction strategies serve various aims, including prediction and control, that are central to understanding the epistemic diversity of systems biology.
\end{abstract}

Systems biology is a new and highly interdisciplinary field that combines elements from molecular biology and physiology with quantitative modelling approaches from disciplines such as engineering, physics, computer science, and mathematics. The term 'systems biology' was used originally in 1968 by Mesarović to urge the use of systems theory to understand biological systems (Mesarović 1968); some commentators would trace the historical roots even further back (Green and Wolkenhauer 2013). But when the term is used in the context of contemporary bioscience it typically refers to a much more recent approach, initiated in the late 1990s as a response to the new experimental techniques and fast computers that allowed the rapid sequencing of DNA and automated measurements of molecular interactions (Ideker et al. 2001; Kitano 2001). These innovations afforded major new initiatives in the life sciences but also produced unforeseen challenges. Systems biology addresses one of these, namely the interpretation of extensive quantitative data via mathematical and computational modelling (Alberghina and Westerhoff 2005; Boogerd et al. 2007).

Research in systems biology is driven by complex problems that require multidisciplinary integration (Carusi 2014; MacLeod and Nersessian 2014; O’Malley and Soyer 2012). Consequently, it is a diverse field. Some proponents pursue strategies that extend molecular biology with sequence-based tools (see Chapter 24), while others explore the relevance of abstract mathematical systems theory to molecular interactions (O’Malley and Dupré 2005). Common to all branches of systems biology is the willingness to borrow reasoning and representation tools from engineering and the physical sciences, including network diagrams and graph-theory, other types of mathematical modelling (primarily ordinary differential equations) and computational simulations. We focus on just one of the many possible questions about systems biology: To what extent can the modelling strategies and explanations in systems biology be characterized as mechanistic? 


\section{Dynamic Mechanistic Explanation and Other Modelling Aims}

A hallmark of mechanistic research is to understand a complex whole by decomposing it into component parts, and by localizing phenomena of interest to certain parts of the system (Bechtel and Richardson 1993; Craver 2007; see Chapters 9 and 19). Models in systems biology are similarly based on empirically measured molecular entities and interactions. Given the abundance of different molecules and pathways in every cell, modelling involves the selection of components relevant to the system being investigated (Donaghy 2014). But the role of mathematical models and computational tools - as distinctive aspects of systems biology — was not addressed in original philosophical accounts of mechanistic explanations (see Chapter 16), primarily because molecular systems biology is so new. Lately, the relationship between models in systems biology and mechanistic accounts has become an important philosophical topic of debate, with some commentators arguing that a traditional mechanistic account is sufficient to describe research in systems biology (e.g., Richardson and Stephan 2007), and others instead stressing the need for a more pluralistic perspective of explanatory integration (e.g., Braillard 2010; Fagan 2016; Mekios 2015).

Although it is possible to focus on differences between dynamic models in systems biology and mechanistic explanations in general (Issad and Malaterre 2015; Théry 2015), Bechtel and Abrahamsen (2010) instead highlight the continuity between the two by introducing the notion of 'dynamic mechanistic explanation.' Dynamic mechanistic explanations are also based on concrete entities and interactions, but they extend mechanistic explanation by mathematically or computationally capturing the dynamical operation of the system and its parts across time. In fact, in the case of complex systems, mathematical models are required for the purpose of mechanistic explanation (Baetu 2015; Bechtel 2012; Brigandt 2013; see Chapter 20). How is this the case?

In addition to decomposition and localization as strategies for discovering mechanism components, mechanistic explanations must reassemble those components and specify epistemically how their organization and operation result in the overall features of the mechanism to be explained (Bechtel and Abrahamsen 2005). Bechtel illustrates the importance of mathematical models with circadian rhythms, which are endogenous oscillations of about 24 hours present in most organisms. A mechanism diagram can depict various components of the underlying mechanism, including specific genes and proteins, some of which have oscillating expression levels. The diagram can also represent the activation or inhibition of interactions among proteins and other entities, thereby depicting positive as well as negative feedback loops (see Chapter 18). For some simple mechanisms, mental simulation (using a mechanism diagram) suffices to show how the phenomenon to be explained is generated (Bechtel and Abrahamsen 2005). However, in the case of circadian rhythms, only mathematical models are able to reveal that over time the component interactions - which involve changing protein concentrations, several feedback loops and time-delaying gene expression pathways - actually produce sustained periodic oscillations (see also Brigandt 2015).

Computational modelling strategies in systems biology can thus extend mechanistic accounts in various ways. By providing mathematical tools for modelling the dynamics of large systems of nonlinear organization, computational models can help researchers recompose knowledge about subsystems that have been taken apart for functional analysis. It is well-known that there is cross-talk between different mechanisms, and computational tools can afford a better understanding of how mechanisms relate to one another dynamically (Bechtel 2015b; Fagan 
2012). Among the many examples are computer simulations of whole cells (and even organs) that explore dynamic interactions between different functional subsystems (Bassingthwaighte et al. 2009; Karr et al. 2012). If a mechanistic explanation is characterized as accounting for how a system behaviour is causally generated by the organized interaction of its particular parts, computational models of circadian rhythms or large-scale simulations can indeed be interpreted as vehicles for mechanistic explanations.

However, we should be careful about assuming that modelling in systems biology is always geared towards mechanistic explanations. First, ethnographic studies of research practices in systems biology show that scientists may not even frame their modelling aims in terms of explanation, but instead, in terms of control or prediction (MacLeod and Nersessian 2015). Practical concerns (application) and pragmatic constraints (not all parameters can be measured and modelled mathematically) direct particular modelling aims. MacLeod and Nersessian make this point with the example of how reducing the amount of cell-wall hardening lignin in plants is highly desirable for biofuel production. But because the relevant model of the lignin synthesis pathway depends on estimated parameters, it indicates primarily a robust relation between particular system components and the lignin output. The model's main achievement, therefore, is to reveal an angle of technological control. Although it does give partial insight into how the system works, this model might not yield a mechanistic explanation in the sense of accounting for the behaviour of the whole in terms of its parts and their properties (MacLeod and Nersessian 2015). Second, even when systems biologists have explanation as an explicit target, they may not be offering causal explanations of specific systems. Instead, they may intend to provide more abstract functional classifications of all the variants of system organization that could possibly realize a particular function (see Section 3).

The lesson we draw from these observations is that while systems biology can fruitfully extend philosophical accounts of mechanistic explanation to include dynamical and quantitative aspects by means of mathematical models and simulations, philosophers investigating systems biology also need to pay attention to the diverse practices across this field, and to the actual, context-dependent aims of the modellers and experimental researchers. We will take this finding into account as we explore mechanisms in systems biology further via the use of network analysis.

\section{Network Models: From Motifs to Global Topologies}

Because cellular systems are highly complex webs of molecular interactions, one approach in systems biology involves the investigation of networks. A network can be represented and studied computationally as a graph, in which the nodes correspond to molecular entities, while an edge between two nodes represents an interaction between them. A graph can be undirected, such as a protein interaction network that depicts all the interactions in which the protein types inside a cell engage, or it can be directed. The latter category includes metabolic reaction networks, signal transduction networks, and gene regulatory networks that depict genes activating other genes.

While decomposition and localization have proven to be useful strategies of mechanistic research, additional methods (e.g., graph-theoretic and computational) are needed in systems biology to process large data sets and analyse highly integrated systems. One particular approach is to screen larger networks for the repeated occurrence of the same type of small connectivity 
patterns called network motifs (Figure 1; Alon 2007). The functionality of any network motif can then be investigated computationally. Consider for example a feedforward loop, in which X has a direct as well as a mediated input on $Z$ (Figure 1A). Using engineering language, systems biologists might say that $Z$ processes its two potential inputs as an AND-gate, which is when both inputs are needed for activation. In this case, the motif will function as a persistence detector. In other words, output $Z$ will be activated only upon sustained activation of $X$, which can be turned on by some external signal. The reason is that when receiving an input by means of the time-delayed pathway via $\mathrm{Y}$ (involving two activation steps), $\mathrm{Z}$ would not receive a second input (directly from X) unless X has already been active for some time. Such a persistencedetector design makes biological sense when it is energetically costly for an organism to synthesize an enzyme that processes a particular substrate. In that case, synthesis is best initiated only if the substrate is reliably present. Particular design motifs are expected to function in the same general way, whatever the particular biological and environmental contexts of their implementation (but see Section 4).

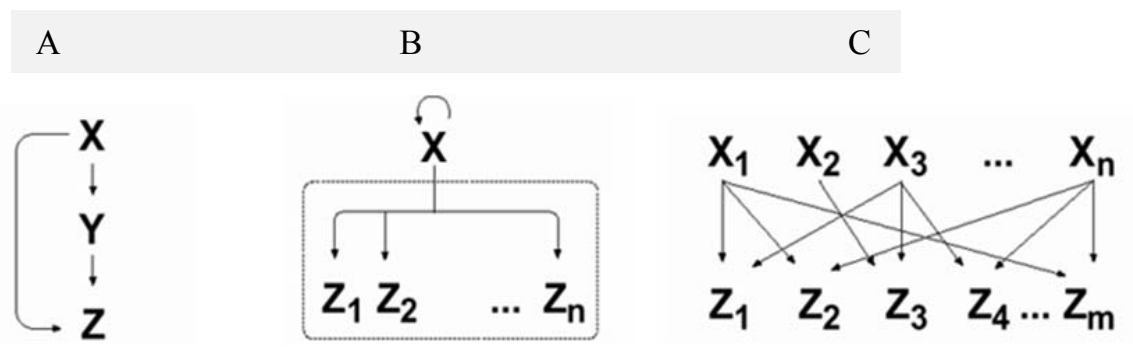

Figure 1. Examples of network motifs. A: Feedforward loop; B: Single-input motif; C: Dense overlapping regulons. Adapted with permission from Alon (2007), copyright Taylor and Francis Group LLC Books.

Network motifs abstract away from a good deal of molecular detail. They neither specify what kinds of entities the nodes are, nor do they indicate the actual means by which one entity would activate another (e.g., that a eukaryotic gene is transcribed to RNA, which when transported outside the nucleus is translated to a protein, which later binds to a different gene so as to activate it). Despite this loss of mechanistic detail, Levy and Bechtel (2013) argue that the analysis of a network motif is still a dynamic mechanistic explanation. This is because once abstracted, the network account points directly to the organization of the mechanism that is responsible for the phenomenon to be explained. Generally, this sort of abstraction occurs widely in systems biological modelling, including the examples already mentioned in Section 1 (see Chapters 17 and 35).

Even though the analysis of an individual network motif's functionality might be largely mechanistic, what makes research on network motifs distinctively systems-biological stems from the fact that large networks are screened to determine the frequency with which motifs occur (e.g., the feedforward loop is known to be highly abundant). Doing so reveals both common and uncommon elements of biological design, and draws attention to the former, which are likely to be more biologically important. Moreover, different kinds of large networks, from gene regulatory to neural networks, can be screened for the same design element. This generalizability also applies to networks from different taxa, whether prokaryotes or eukaryotes. These strategies indicate that abstract organizational schemes, which systems biologists call design principles, transcend the organization of a single mechanism, and even a single species (Green 2015b). We 
will elaborate on design principles in Section 3.

While the scrutiny of an individual motif pertains to a very small network, research at the other end of the spectrum investigates large networks for their global properties, also via graphtheoretical means. Earlier work initially addressed regular networks (where each node has the same number of edges) and random networks (where a certain proportion of nodes is randomly connected by edges; Figure 2, left). In the last fifteen years, however, small-world and scale-free networks have gained prominence due to their interesting properties and widespread occurrence among real biological systems. A small-world network is defined in terms of the global property of the average path length between two nodes - averaged across all pairs of the network's nodes - which grows logarithmically as the number of nodes increases. This means that for two randomly chosen nodes, the shortest distance between them (in terms of a path of intermediate nodes connected by edges) will be small relative to the size of the network. This global property usually entails that a signal propagates quickly from one part of the network to another. For a biological system this can have the advantage of enabling rapid reaction times. Many proteininteraction networks are small-world for this reason (Albert 2005).

A network's degree distribution $P(k)$ is the network-wide proportion of edges that is connected to $k$ other nodes (i.e., the network's proportion of nodes connected to only one other node, the proportion of nodes connected to two other nodes, and so on). The degree distribution is thus a global characteristic. A scale-free network is defined as a network that has a degree distribution that follows a power law of the form $P(k)=c \cdot k^{-\gamma}$. This exponentially declining function means that across any scale-free network there are many nodes that are connected to only one or a few other nodes, while only few nodes are so-called $h u b s$, which are connected to many other nodes (Figure 2, right). From this global property, predictions can be made about the network's functionality. One is that it will exhibit robustness, which is the biologically important feature that a system will maintain its functionality despite perturbations. While the elimination of a node that is connected to one, or only a few other nodes, is unlikely to affect a network's functionality, eliminating a node that is a hub may seriously impact how the network functions. But in a scale-free network there are comparatively few hubs, meaning that such a network is generally robust. A variety of actual biological networks of interest to systems biology are approximately scale-free, including metabolic networks and the gene regulatory networks of prokaryotes and eukaryotes (Albert 2005). 
A Random network

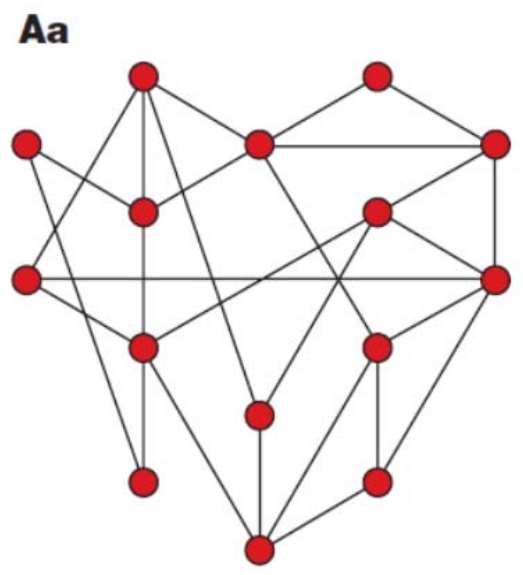

B Scale-free network

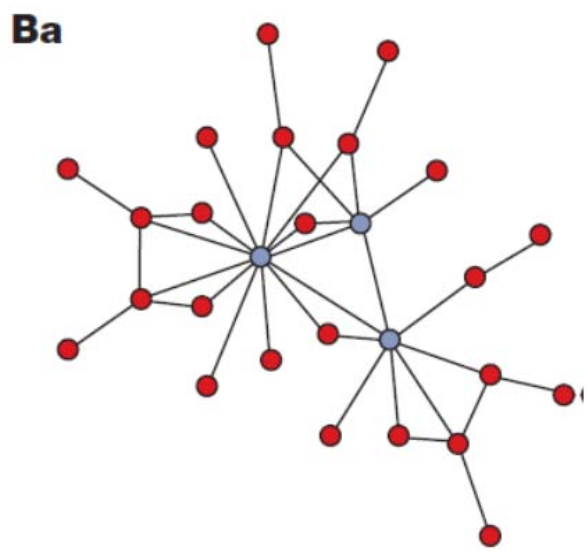

Figure 2. An illustration of two kinds of large-scale networks. In the scale-free network, highly connected hub nodes are visualized in lighter grey. Reprinted from Barabási and Oltvai (2004) with permission from Nature Reviews Genetics, Macmillan Publishers Ltd, copyright 2004.

Huneman (2010) coined the term 'topological explanation' for explanations of phenomena in terms of topological properties (including the structural properties of a graph). Although his focus is on ecological systems and evolutionary contexts, one important explanandum he addresses is equally relevant in systems biology: namely, robustness. Huneman argues that an explanation of a system's general robustness to random node elimination in terms of its scalefree network structure is a topological explanation. A topological explanation appeals to a system's basic organization, in the same way the network motif explanations mentioned above do (Levy and Bechtel 2013), but it abstracts away from even the generic interactions or temporal features seen in motifs. This is at odds with mechanistic explanation, if the latter is to include significant physical detail (Craver 2007; Kaplan and Craver 2011), or if a mechanistic account's explanatory status is taken to increase when more detail is added (Kaplan 2011; see Chapter 20). In any case, the fact that topological explanations neither list specific activities nor trace their operation from set-up to termination conditions is Huneman's primary reason for contrasting this type of explanation against mechanistic explanation.

Another case of topological explanation in the context of systems biology is the explanation of vulnerability (the opposite of robustness) in terms of bowtie structure (Jones 2014). A bowtie structure is a molecular network with the shape of a bowtie (Figure 3), in which it is obvious that the bowtie's core is the weakest link because its deactivation (compared to any other node) will probably damage the whole network's functionality. A concrete example is the explanation of why the human immune system is vulnerable to attacks on CD4+ T-cells (by HIV). The reason is that the molecular network of intercellular interactions and signalling pathways forms a bowtie that has the CD4+ T-cell type as its core (Figure 3; Kitano and Oda 2006). Generally then, it holds for scale-free networks that they are robust to random perturbations but vulnerable to attacks on the highly connected nodes (hubs or bowtie cores) that participate in a large number of interactions. Topological explanations such as these may well be instances of what some philosophers have discussed as distinctively mathematical explanations in natural science, which 
have even been claimed to offer non-causal explanations of physical phenomena (Lange 2013). Our reason for invoking topological explanation, however, is simply to show how it contrasts with classic mechanistic accounts (see Bechtel 2015a; Woodward 2013).

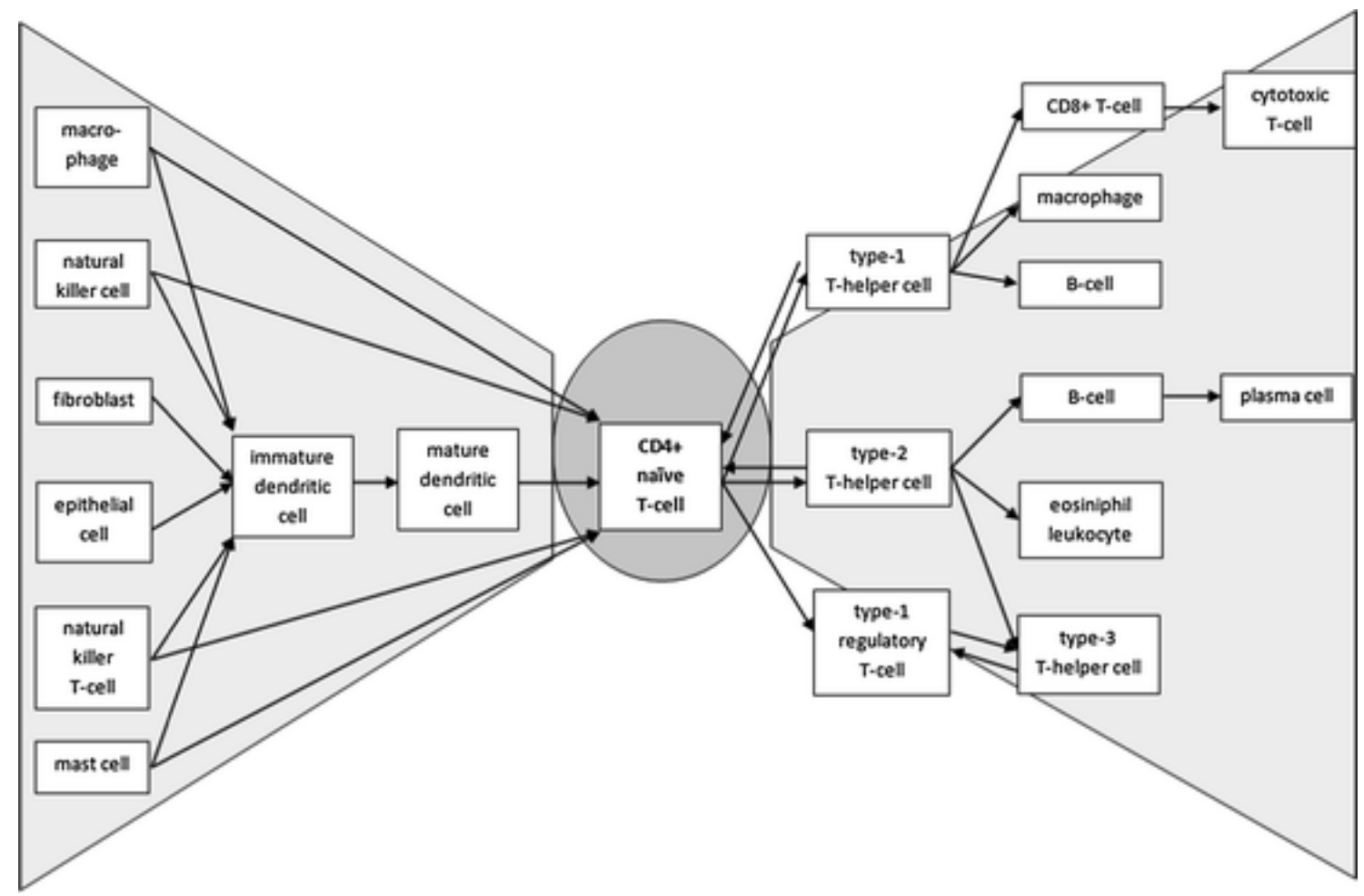

Figure 3: Bowtie network of CD4+ T-cells. Reproduced from Jones (2014) with permission from Erkenntnis, Springer, copyright 2014.

Despite these insights, the value of graph-theoretical analysis for biological research is a contested issue, and critics have pointed to problems with the generalizations made about such networks (Arita 2004). For instance, whether gene regulatory networks are scale-free has been disputed, because some biological networks also exhibit properties similar to random networks (Barabási and Oltvai 2004; Keller 2005). Another common challenge is that networks usually provide a static picture of cellular systems, because the data that network edges are based on combine the totality of interactions that have been measured. However, all the edges represented need not be active at the same time or in the same location in vivo. A recent development, therefore, is to include temporal change when constructing topological mappings. When timecourse data is used, distinctions can be made between a 'party hub', which interacts with many entities at the same time, and a 'date hub', which interacts with only a few other entities despite having many overall connections and interaction partners (Han et al. 2004). In yeast metabolism research, protein interaction and gene expression data has been used to develop a time-dependent network that is sensitive to which proteins interact at a particular phase of the cell cycle (de Lichtenberg et al. 2005). This has provided new insight into the processes underlying the periodization of protein synthesis.

As Section 1 discussed, research methods in systems biology have huge potential not only for extending mechanistic research but also for providing novel insights into how and why biological systems are organized into generalizable schemes with broad applications. As we have 
demonstrated, graph-theoretical analysis affords a quantitative understanding of biological function and makes possible a comparison of organizational schemes in different functional systems. As well as cellular systems, neuronal, ecological, and even non-biological communication and transport networks are often scale-free or small-world networks, and can be analysed accordingly. Now we will show how network and systems analysis can be taken even further epistemically, in a way that provides additional philosophical insight into the relationship between systems biology and mechanistic research.

\section{Searching for and Using Design Principles}

An important research question in systems biology is the extent to which biological functions rely on general design principles that are largely independent of specific causal details and particular contexts of implementation (Poyatos 2012). Design principles are abstractions that describe characteristic organizational features of importance for a system's functionality, such as negative feedback control, network motif configurations, or common architectures of biological and engineered networks. Aside from understanding how these design principles are causally instantiated in specific biological systems, an important explanatory question is why the same basic principles can describe the functioning of so many different systems. Some philosophers have recently argued that certain abstract models in systems biology, when answering that question, provide non-mechanistic design explanations that focus on generalizable constraints for biological functions (Braillard 2010; Green 2015b). In contrast, discussions of mechanisms have typically interpreted abstract models solely as heuristic tools or as mechanism schemas that guide the formulation of more realistic models (Matthiessen 2015; see Chapter 19). This resonates with the perspective of many experimental biologists, but has long been opposed by proponents of systems theory (Green and Wolkenhauer 2013). Rather than assuming that a model is useful only insofar it explains a biological system in concrete detail, current philosophical investigations of design explanations (and of topological explanations) are motivated by the goal of making sense of why some scientists rely on abstract models even in situations where more detailed models exist.

One illuminating example is how biologists investigate systems exhibiting robust perfect adaptation (RPA) from an engineering perspective. RPA is the capacity of a system with sensors to return to the exact pre-stimulus activity after a stimulus-response reaction. This is important because it maintains the responsiveness of sensors. Creating designs with RPA is a goal in engineering human-made systems. Biological systems also exhibit RPA. Examples include the regulation of calcium homeostasis in mammals and membrane turgor pressure in yeast (Briat et al. 2015). In bacterial chemotaxis (movement in response to external chemical stimuli), RPA pertains to the regained responsiveness of transmembrane receptors (i.e., sensors) that detect changes in the concentration of chemicals in the environment. Adding a repellent to the bacterial environment leads to changes in the bacterial tumbling frequency (and thereby to random reorientations in space), but the receptor system returns very quickly to its equilibrium value. This enables the receptors to become sensitive to new changes, even if the repellent concentration continues increasing, which occurs when the bacterium swims along a chemical gradient. In the case of the bacterium $E$. coli, the mechanistic basis of its chemotactic RPA is known. It consists of transmembrane receptors, a signal transduction pathway inside the bacterium, and its connection to the flagellar motor. A feedback loop from the intracellular process back to the transmembrane receptor is important for achieving RPA (Barkai and Leibler 
1997).

The explanatory issue we are highlighting with bacterial chemotaxis is the question of what generic properties (abstract organizational features) make it possible for any system - not just $E$. coli - to exhibit RPA. The answer is integral feedback control (Yi et al. 2000). Used in engineering, integral feedback control is known in mathematical control theory as a special case of the internal model principle. When the environmental input $u$ changes (see Figure 4), the difference between the actual output $y_{1}$ and the desired output $y_{0}$ - the equilibrium value of the receptor - is fed back into the system as the integral of the system error. This feedback functions as a signal for the renormalization of the receptor, so that integral feedback control is sufficient for RPA. A crucial insight is provided by a theorem of Yi et al. (2000), which shows that (at least in linear systems) integral feedback control is necessary for achieving RPA. This explains why any system that exhibits RPA has to have an organization that instantiates integral feedback control (Iglesias 2013). E. coli should be no exception in this regard, and Yi et al. (2000) point out that an influential dynamic mechanistic model of RPA in bacteria (Barkai and Leibler 1997) does indeed embody the basic principle of integral feedback control.

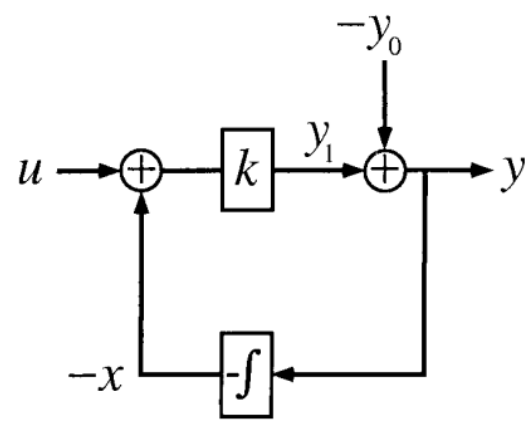

\begin{tabular}{|c|c|}
\hline $\begin{aligned} \dot{x} & =y \\
y & =y_{1}-y_{0} \\
& =k(u-x)-y_{0}\end{aligned}$ & $\begin{array}{l}y(t) \rightarrow 0 \text { as } t \rightarrow 0 \\
\text { iff } \\
k>0\end{array}$ \\
\hline
\end{tabular}

Figure 4. Diagram showing the abstract principle of Integral Feedback Control. Reproduced from Yi et al. (2000), with permission from PNAS, copyright (2000) National Academy of Sciences,

USA.

We can compare this account of chemotaxis with a standard mechanistic explanation. The latter would show how a particular structural organization causally generates and thus explains some function (e.g., RPA). In contrast, what Wouters (2007) calls a design explanation proceeds in the opposite direction, as the function to be performed (RPA in our case) explains the presence of some structural organization (integral feedback control). Using examples from physiology and functional anatomy, Wouters argues that such an explanation is non-causal, because it is based on law-like dependency relations between structures and functions. It maps out the possible structural realizers of a certain function, without going into a diachronic account of how the realizer or the need for the function came about causally. But regardless of where one stands on the status of non-causal explanations, in the case of bacterial chemotaxis, the design explanation does not just offer a list of the various concrete mechanisms that perform RPA (e.g., a transmembrane receptor, six Che proteins, and other details in E. coli). This is a non- 
mechanistic explanation in that it points to the abstract organizational feature of integral feedback control as a generic property that any system exhibiting RPA must instantiate (Braillard 2010). This explanatory aim addresses a why-question that is distinct from the aim of explaining how a behaviour is mechanistically produced in some specific system.

The example of design principles underpinning RPA in engineering and biology also provides more general philosophical lessons about the theoretical relevance of delineating the space of biological possibility. Mechanistic accounts have typically taken how-possibly models to have less explanatory power than how-actually models (Craver 2007; Kaplan 2011; Kaplan and Craver 2011; see Chapter 19). Yet, understanding the wider constraints on biological variation can in some contexts be of higher importance than describing how a specific function is causally produced in any specific system. Design principles can help researchers understand why the same structural patterns are found across different contexts: as a result of the constraints on possible architectures that can realize a given function. Importantly, this is not to be understood as a question that presupposes natural selection as the answer. Rather, the why-question addressed here is about the physically determined boundaries of the design space for a given function.

Design principles do, however, have significance for evolutionary research as well as functional biology. Investigations of the constraints on evolutionary and developmental trajectories have often been associated with rather speculative 'structuralist' accounts, but some of these ideas have gained new relevance in the context of evolutionary systems biology (Green et al. 2015; Jaeger and Crombach 2012). Evolutionary systems biology is an umbrella term for very diverse approaches (O’Malley 2012), but one important aim is to investigate why certain general patterns arise in evolution. This is often done via models that represent the in silico evolution of gene regulatory networks. Structures like the network motifs discussed in Section 2 are often assumed to be common because of regulatory functions favoured by natural selection (Alon 2007). Yet evolutionary simulation studies suggest that common structural patterns of networks, such as feedforward loops, may also result from constraints on genome evolution. These constraints are inherent in the mutational dynamics of gene duplication, deletion and recombination (Cordero and Hogeweg 2006). Research on evolutionary design principles, when understood as general patterns occurring from evolutionary trajectories, can thus generate insight into the potential and limits of biological variation.

Design principles also identify the generic features that unite diverse systems exhibiting similar functional patterns (Green 2015b). By relating specific systems to general functional types, such as signal amplifiers, filters, or homeostatic regulators, these abstract principles facilitate the transfer of theoretical frameworks across disciplinary borders. Aside from this epistemic role, such structure-function mappings can serve practical aims. Similarly to Macleod and Nersessian's (2015) emphasis on practical purposes such as control and prediction of modelling in systems biology, research on possibility spaces for biological structures can have practical goals such as templates for synthetic biology designs. Synthetic biology is the biological construction of material models, usually guided by mathematical modelling. 'Howpossibly models' can in this context be more important than 'how-actually models' because they elucidate the necessary structures for a certain function, like RPA, or reveal whether there are simpler possible designs than the ones found in nature (Briat et al. 2015; Ma et al. 2009).

The upshot of this discussion is that abstract models are not always stepping stones toward more detailed mechanistic models. Aside from the practical purposes of control and 
technological implementation, abstract design principles afford an understanding of why causally different systems in biology and engineering share certain organizational features, and how they are situated within larger spaces of physically possible designs. Consequently, an exclusive philosophical focus on mechanistic explanation (and even on dynamic mechanistic explanation) risks missing out on these diverse epistemic activities in systems biology.

\section{Discussion and Outlook}

Research in systems biology shows how strategies of abstraction are used in biology not only to simplify the task of identifying mechanisms but also to elucidate system-level patterns of organization that may not be visible at the level of the molecular details. Mechanistic accounts have usually been framed in opposition to explanatory unification, understood as the subsumption of the particular to general laws or explanatory schemas. But network modelling and the quest for design principles suggest an alternative way of thinking about the role of unification in biology: not via reduction of the particular to the general, but through abstraction from causal details for the purpose of identifying generic organizational patterns.

Mathematical models (including network models and design principles) serve various roles in systems biology. Generally, mathematical frameworks provide a more rigorous way of exploring the extent to which biological functions are underpinned by characteristic organizational structures. Mathematical frameworks can also make engineering analogies more precise. Section 2 mentioned the identification of functional network motifs based on mathematically guided screening for overabundant circuit types. This search is inspired by an analogy to design principles in electronic networks, and the structural decomposition of the network preceded the functional analysis of the modules of the network (see Chapter 35). In other cases, the biological function is known and systems biologists set out to explore the extent to which the function is similarly realized in engineered systems (e.g., robustness). Mathematical abstractions and design principles can articulate constraints that delimit the search space for an analysis. Delineating search space may serve the development of mechanistic explanations. At the same time, network models and design principles provide an understanding complementary to mechanistic explanation. Although an important virtue of mechanistic explanations is to make sense of biological diversity through attention to specific causal difference-makers and material composition, abstraction strategies can help scientists see similarities in the way functional systems - from airplanes to organisms - are organized.

Generally, a focus by philosophers on the issue of mechanistic explanation has left many aspects of systems biology unexplored. We have pointed to the use of models for the purpose of prediction, control, or the creation of simple and efficient designs that can be implemented in synthetic organisms. Another topic of major interest to systems biologists that is philosophically rich is robustness. In many cases when a system maintains its functioning despite noise and even major perturbations, this is due to dynamic reorganization, where the organismal system responds flexibly by changing interaction patterns and levels, including establishing new interactions (Wagner 2005). This puts pressure on the assumption that systems biology always investigates mechanisms (on a machine-like conception), or that all explanations about systems exhibiting dynamic reorganization or robustness are mechanistic (in the sense of referencing the mechanism's specific organization; Brigandt 2015; Gross 2015; Woodward 2013).

Some of the questions that deserve more attention by philosophers pertain to issues that are 
currently controversial within the systems biology community itself. One is the question of whether complex living systems can be understood in terms of engineering notions (Braillard 2015; Green 2015a), and particularly whether the heuristic assumption of modularity is warranted. Research on network motifs is often predicated on the idea that an individual motif is modular, meaning that its functionality is unaffected by the system context in which it occurs (Section 2). The traditional mechanistic strategies of decomposition into distinct components and the localization of some function to a certain component also resonate with the assumption that biological systems are modular. However, many systems biologists observe highly integrated functionality across large-scale networks, which suggests that systems need to be investigated not in terms of modularity but via more connectivist perspectives that can capture features emerging from system-wide dynamics (Huang 2004; see also Bassingthwaighte et al. 2009; Bechtel 2015a).

Our discussion draws attention to a wide range of explanatory and modelling strategies in systems biology. We have shown how some explanatory aims and outputs are not mechanistic according standard philosophical interpretations of mechanistic explanations, and indeed, that some of the practices in systems biology lie outside existing philosophical frameworks. But well beyond these negative insights, we have depicted the wealth of modelling approaches at work in systems biology, and how further philosophical scrutiny of them will enhance the investigation of biological systems and philosophical accounts of mechanistic explanation and explanation in general.

\section{References}

Alberghina, L. and H. V. Westerhoff (eds), (2005) Systems Biology: Definitions and Perspectives, Berlin: Springer.

Albert, R. (2005) "Scale-Free Networks in Cell Biology," Journal of Cell Science 118: 4947-57. Alon, U. (2007) An Introduction to Systems Biology: Design Principles of Biological Circuits, Boca Raton: Chapman \& Hall / CRC Press.

Arita, M. (2004) “The Metabolic World of Escherichia coli Is Not Small," Proceedings of the National Academy of Sciences USA 101: 1543-7.

Baetu, T. (2015) "From Mechanisms to Mathematical Models and Back to Mechanisms: Quantitative Mechanistic Explanations," in P.-A. Braillard and C. Malaterre (eds.), Explanation in Biology: An Enquiry into the Diversity of Explanatory Patterns in the Life Sciences, Dordrecht: Springer, pp. 345-63.

Barabási, A.-L. and Z. N. Oltvai (2004) "Network Biology: Understanding the Cell's Functional Organization," Nature Reviews Genetics 5: 101-13.

Barkai, N. and S. Leibler (1997) "Robustness in Simple Biochemical Networks," Nature 387: 913-7.

Bassingthwaighte, J., P. Hunter and D. Noble (2009) "The Cardiac Physiome: Perspectives for the Future," Experimental Physiology 94: 597-605.

Bechtel, W. (2012) "Understanding Endogenously Active Mechanisms: A Scientific and Philosophical Challenge," European Journal for Philosophy of Science 2: 233-48. - (2015a) "Can Mechanistic Explanation Be Reconciled with Scale-Free Constitution and Dynamics?" Studies in History and Philosophy of Biological and Biomedical Sciences 53: 84-93. (2015b) "Using Computational Models to Discover and Understand Mechanisms," 
Studies in History and Philosophy of Science, doi:10.1016/j.shpsa.2015.10.004.

Bechtel, W. and A. Abrahamsen (2005) "Explanation: A Mechanist Alternative," Studies in History and Philosophy of Biological and Biomedical Sciences 36: 421-41.

- (2010) "Dynamic Mechanistic Explanation: Computational Modeling of Circadian Rhythms as an Exemplar for Cognitive Science," Studies in History and Philosophy of Science 41: 321-33.

Bechtel, W. and R. C. Richardson (1993) Discovering Complexity: Decomposition and Localization as Strategies in Scientific Research, Princeton: Princeton University Press.

Boogerd, F. C., F. J. Bruggeman, J.-H. S. Hofmeyr and H. V. Westerhoff (eds), (2007) Systems Biology: Philosophical Foundations, Amsterdam: Elsevier.

Braillard, P.-A. (2010) "Systems Biology and the Mechanistic Framework," History and Philosophy of the Life Sciences 32: 43-62. (2015) "Prospect and Limits of Explaining Biological Systems in Engineering Terms," in P.-A. Braillard and C. Malaterre (eds.), Explanation in Biology: An Enquiry into the Diversity of Explanatory Patterns in the Life Sciences, Dordrecht: Springer, pp. 319-44.

Briat, C., A. Gupta and M. Khammash (2015) "A New Motif for Robust Perfect Adaptation in Noisy Biomolecular Networks," arXiv.org e-Print Archive arXiv:1410.6064v4 [math.OC]. Brigandt, I. (2013) "Systems Biology and the Integration of Mechanistic Explanation and Mathematical Explanation," Studies in History and Philosophy of Biological and Biomedical Sciences 44: 477-92.

- (2015) "Evolutionary Developmental Biology and the Limits of Philosophical Accounts of Mechanistic Explanation," in P.-A. Braillard and C. Malaterre (eds.), Explanation in Biology: An Enquiry into the Diversity of Explanatory Patterns in the Life Sciences, Dordrecht: Springer, pp. 135-73.

Carusi, A. (2014) "Validation and Variability: Dual Challenges on the Path from Systems Biology to Systems Medicine," Studies in History and Philosophy of Biological and Biomedical Sciences 48: 28-37.

Cordero, O. X. and P. Hogeweg (2006) "Feed-Forward Loop Circuits as a Side Effect of Genome Evolution," Molecular Biology and Evolution 23: 1931-6.

Craver, C. F. (2007) Explaining the Brain: Mechanisms and the Mosaic Unity of Neuroscience, Oxford: Oxford University Press.

de Lichtenberg, U., L. J. Jensen, S. Brunak and P. Bork (2005) "Dynamic Complex Formation

During the Yeast Cell Cycle," Science 307: 724-7.

Donaghy, J. (2014) "Temporal Decomposition: A Strategy for Building Mathematical Models of Complex Metabolic Systems," Studies in History and Philosophy of Biological and Biomedical Sciences 48: 1-11.

Fagan, M. B. (2012) "Waddington Redux: Models and Explanation in Stem Cell and Systems Biology," Biology \& Philosophy 27: 179-213.

(2016) "Stem Cells and Systems Models: Clashing Views of Explanation," Synthese 193: 873-907.

Green, S. (2015a) "Can Biological Complexity Be Reverse Engineered?" Studies in History and Philosophy of Biological and Biomedical Sciences 53: 73-83. (2015b) "Revisiting Generality in Biology: Systems Biology and the Quest for Design Principles," Biology \& Philosophy 30: 629-52.

Green, S., M. Fagan and J. Jaeger (2015) "Explanatory Integration Challenges in Evolutionary Systems Biology," Biological Theory 10: 18-35.

Green, S. and O. Wolkenhauer (2013) “Tracing Organizing Principles: Learning from the 
History of Systems Biology," History and Philosophy of the Life Sciences 35: 553-76.

Gross, F. (2015) "The Relevance of Irrelevance: Explanation in Systems Biology," in P.-A. Braillard and C. Malaterre (eds.), Explanation in Biology: An Enquiry into the Diversity of Explanatory Patterns in the Life Sciences, Dordrecht: Springer, pp. 175-98.

Han, J.-D. J., N. Bertin, T. Hao, D. S. Goldberg, G. F. Berriz, L. V. Zhang, D. Dupuy, et al. (2004) "Evidence for Dynamically Organized Modularity in the Yeast Protein-Protein Interaction Network," Nature 430: 88-93.

Huang, S. (2004) "Back to the Biology in Systems Biology: What Can We Learn from Biomolecular Networks?" Briefings in Functional Genomics and Proteomics 2: 279-97. Huneman, P. (2010) "Topological Explanations and Robustness in Biological Sciences," Synthese 177: 213-45.

Ideker, T., T. Galitski and L. Hood (2001) “A New Approach to Decoding Life: Systems Biology," Annual Review of Genomics and Human Genetics 2: 343-72.

Iglesias, P. (2013) "Systems Biology: The Role of Engineering in the Reverse Engineering of Biological Signaling," Cells 2: 393-413.

Issad, T. and C. Malaterre (2015) "Are Dynamic Mechanistic Explanations Still Mechanistic?" in P.-A. Braillard and C. Malaterre (eds.), Explanation in Biology: An Enquiry into the Diversity of Explanatory Patterns in the Life Sciences, Dordrecht: Springer, pp. 265-92.

Jaeger, J. and A. Crombach (2012) "Life's Attractors: Understanding Developmental Systems through Reverse Engineering and in Silico Evolution," in O. S. Soyer (ed.), Evolutionary Systems Biology, New York: Springer, pp. 93-119.

Jones, N. (2014) "Bowtie Structures, Pathway Diagrams, and Topological Explanation," Erkenntnis 79: 1135-55.

Kaplan, D. M. (2011) "Explanation and Description in Computational Neuroscience," Synthese 183: 339-73.

Kaplan, D. M. and C. F. Craver (2011) "The Explanatory Force of Dynamical and Mathematical Models in Neuroscience: A Mechanistic Perspective," Philosophy of Science 78: 601-27.

Karr, J. R., J. C. Sanghvi, D. N. Macklin, M. V. Gutschow, J. M. Jacobs, B. Bolival, N. AssadGarcia, J. I. Glass and M. W. Covert (2012) "A Whole-Cell Computational Model Predicts Phenotype from Genotype," Cell 150: 389-401.

Keller, E. F. (2005) “Revisiting "Scale-Free" Networks,” BioEssays 27: 1060-8.

Kitano, H. (ed), (2001) Foundations of Systems Biology, Cambridge, MA: MIT Press.

Kitano, H. and K. Oda (2006) "Robustness Trade-Offs and Host-Microbial Symbiosis in the Immune System," Molecular Systems Biology 2.

Lange, M. (2013) "What Makes a Scientific Explanation Distinctively Mathematical?" British Journal for the Philosophy of Science 64: 485-511.

Levy, A. and W. Bechtel (2013) "Abstraction and the Organization of Mechanisms," Philosophy of Science 80: 241-61.

Ma, W., A. Trusina, H. El-Samad, W. A. Lim and C. Tang (2009) "Defining Network Topologies That Can Achieve Biochemical Adaptation," Cell 138: 760-73.

MacLeod, M. and N. J. Nersessian (2014) "Strategies for Coordinating Experimentation and Modeling in Integrative Systems Biology," Journal of Experimental Zoology Part B: Molecular and Developmental Evolution 322: 230-9.

MacLeod, M. and N. J. Nersessian (2015) "Modeling Systems-Level Dynamics: Understanding without Mechanistic Explanation in Integrative Systems Biology," Studies in History and Philosophy of Biological and Biomedical Sciences 49: 1-11.

Matthiessen, D. (2015) "Mechanistic Explanation in Systems Biology: Cellular Networks," 
British Journal for the Philosophy of Science, doi:10.1093/bjps/axv011.

Mekios, C. (2015) "Explanation in Systems Biology: Is It All About Mechanisms?" in P.-A.

Braillard and C. Malaterre (eds.), Explanation in Biology: An Enquiry into the Diversity of Explanatory Patterns in the Life Sciences, Dordrecht: Springer, pp. 41-72.

Mesarović, M. D. (1968) "Systems Theory and Biology_-View of a Theoretician," in M. D. Mesarović (ed.), Systems Theory and Biology: Proceedings of the III Systems Symposium at Case Institute of Technology, Berlin: Springer, pp. 59-87.

O’Malley, M. A. (2012) "Evolutionary Systems Biology: Historical and Philosophical Perspectives on an Emerging Synthesis," in O. S. Soyer (ed.), Evolutionary Systems Biology, New York: Springer, pp. 1-28.

O’Malley, M. A. and J. Dupré (2005) "Fundamental Issues in Systems Biology," BioEssays 27: 1270-6.

O’Malley, M. A. and O. S. Soyer (2012) "The Roles of Integration in Molecular Systems

Biology," Studies in History and Philosophy of Biological and Biomedical Sciences 43: 5868.

Poyatos, J. (2012) "On the Search for Design Principles in Biological Systems," in O. S. Soyer (ed.), Evolutionary Systems Biology, New York: Springer, pp. 183-93.

Richardson, R. C. and A. Stephan (2007) "Mechanism and Mechanical Explanation in Systems Biology," in F. C. Boogerd, F. J. Bruggeman, J.-H. S. Hofmeyr and H. V. Westerhoff (eds.), Systems Biology: Philosophical Foundations, Amsterdam: Elsevier, pp. 123-44.

Théry, F. (2015) "Explaining in Contemporary Molecular Biology: Beyond Mechanisms," in P.A. Braillard and C. Malaterre (eds.), Explanation in Biology: An Enquiry into the Diversity of Explanatory Patterns in the Life Sciences, Dordrecht: Springer, pp. 113-33.

Wagner, A. (2005) Robustness and Evolvability in Living Systems, Princeton: Princeton University Press.

Woodward, J. (2013) "Mechanistic Explanation: Its Scope and Limits," Aristotelian Society Supplementary Volume 87: 39-65.

Wouters, A. (2007) "Design Explanation: Determining the Constraints on What Can Be Alive," Erkenntnis 67: 65-80.

Yi, T.-M., Y. Huang, M. I. Simon and J. Doyle (2000) "Robust Perfect Adaptation in Bacterial Chemotaxis through Integral Feedback Control," Proceedings of the National Academy of Sciences USA 97: 4649-53. 